\title{
Equivalent Mass versus Life Cycle Cost for Life Support Technology Selection
}

\author{
Harry Jones \\ NASA Ames Research Center
}

Copyright $\odot$ 2003Society of Automotive Engineers, Inc.

\begin{abstract}
The decision to develop a particular life support technology or to select it for flight usually depends on the cost to develop and fly it. Other criteria such as performance, safety, reliability, crew time, and technical and schedule risk are considered, but cost is always an important factor. Because launch cost would account for much of the cost of a future planetary mission, and because launch cost is directly proportional to the mass launched, equivalent mass has been used instead of cost to select advanced life support technology. The equivalent mass of a life support system includes the estimated mass of the hardware and of the spacecraft pressurized volume, power supply, and cooling system that the hardware requires. The equivalent mass of a system is defined as the total payload launch mass needed to provide and support the system. An extension of equivalent mass, Equivalent System Mass (ESM), has been established for use in the Advanced Life Support project. ESM adds a mass-equivalent of crew time and possibly other cost factors to equivalent mass. Traditional equivalent mass is strictly based on flown mass and reflects only the launch cost. ESM includes other important cost factors, but it complicates the simple "flown mass" definition of equivalent mass by adding a non-physical mass penalty for crew time that may exceed the actual flown mass. Equivalent mass is used only in life support analysis. Life Cycle Cost (LCC) is much more commonly used. LCC includes DDT\&E, launch, and operations costs. For Earth orbit rather than planetary missions, the launch cost is less than the cost of Design, Development, Test, and Evaluation (DDT\&E). LCC is a more inclusive cost estimator than equivalent mass. The relative costs of development, launch, and operations vary depending on the mission destination and duration. Since DDT\&E or operations may cost more than launch, LCC gives a more accurate relative cost ranking than equivalent mass. To select the lowest cost technology for a particular application we should use LCC rather than equivalent mass.
\end{abstract}

\section{INTRODUCTION}

This paper begins by discussing the role of cost in system design and technology selection and then describes the use of equivalent mass as a proxy for launch cost. Then the three major costs that comprise Life Cycle Cost (LCC) are described. These are DDT\&E (Design, Development, Test, and Engineering), launch and emplacement, and operations costs.

The following sections of this paper are:

1. Cost in system design

2. Equivalent mass

3. ESM (Equivalent System Mass)

4. Application of equivalent mass

5. DDT\&E (Design, Development, Test, and Engineering) cost

6. Launch and emplacement cost

7. Operations cost, and,

8. Life Cycle Cost (LCC)

These sections are followed by a conclusion. Two appendices consider specialized topics, the definitions of equivalent mass and ESM and the equivalent mass of crew time

\section{COST IN SYSTEM DESIGN}

Cost is a critical decision factor in designing life support systems for human space missions. Cost affects the technology selection, the system design, and the overall mission architecture. Life support cost is a large part of the total mission cost for long duration missions. The extremely high cost of human space flight is a major problem in developing future missions.

Cost is sometimes the only discriminating factor in a design decision. Suppose that the life support system requirements are clearly defined and can be met by several different designs. We then should simply select the least costly design alternative. In this ideal situation, minimizing the expected cost is all that is necessary. 
Unfortunately, system requirements are sometimes poorly defined, or changing, or difficult to achieve with acceptable cost. And even when the requirements are solid, different designs often perform their intended functions more or less well, or have accompanying benefits or penalties. How can we compare systems that do not meet the same requirements in exactly the same way?

BENEFIT/COST RATIO - The merit or cost-effectiveness of dissimilar systems can be weighed using the benefitto-cost ratio. (Larson and Balogh 2000, p. 51)

Merit $=$ benefit/cost

If the different systems can not be scaled to have equal functional performance, perhaps because they are already in final flight packaged form, the benefit might be the quantity of the desired life support product. For example, the benefit might be oxygen generating capacity. In other cases, the system benefit might be a complex figure of merit combining functional performance with other factors such as flexibility or commonality. Many different metrics have been used to evaluate life support systems. (Jones 1999)

RISK - Every system has some risk of failure. Any shortcoming in providing vital life support functions is extremely serious, and life support designers do not intentionally accept a significant risk of failure. But some life support equipment may provide amenities or only perform monitoring or experiments. In such cases, some non-negligible risk of failure may be acceptable to save cost. In this case, the system benefit should be weighted by the probability of success in providing the benefit. (Larson and Balogh 2000, p. 51)

Merit $=\left(\right.$ probability of success ${ }^{*}$ benefit $) /$ cost

The merit or cost-effectiveness might be elaborated to include several different benefits with different probabilities of success. Similarly, the estimated cost might be replaced by its expected value over different system development and mission scenarios. Risk analysis is an important aspect of system design. (Jones 2003)

The above simple formula for system merit manages to capture the key factors in system design. Costs and benefits must be traded off while considering risk. Cost alone is not a sufficient basis for design decisions. Benefits and risks must be explicitly considered.

COST AND MASS - Cost is an extremely important design factor, but cost is difficult to predict. Preliminary cost estimates are often highly inaccurate, due to intrinsic imprecision of the methods, to unanticipated future events, or to excessive optimism. Because of the difficulties using cost, early project design decisions are often based on estimated mass rather than estimated cost. Mass is a more objective physical quantity than cost. Mass directly determines launch cost. Development cost models are usually based on mass. We next consider equivalent mass as a cost metric.

\section{EQUIVALENT MASS}

DEFINITION - The equivalent mass of a system is the sum of the estimated mass of the hardware, of its required materials and spares, and of the pressurized volume, power supply, and cooling system needed to support the hardware in space. Equivalent mass is the total launch mass needed to provide the system function. Equivalent mass directly determines the launch cost. Reducing equivalent mass reduces launch cost, which is a large portion of the total cost of a planetary mission.

Equivalent mass has been used in life support analysis since the Apollo program. It is well established and apparently undisputed that equivalent mass should include mass, volume, power, cooling, and materials and spares logistics. Traditional equivalent mass includes no other factors, but in 1992 Drysdale and his coauthors added a new factor, the crew time needed to support operations and maintenance of the hardware. (Drysdale et al. 1992) This expanded equivalent mass was renamed Equivalent System Mass (ESM) in 1998, when ESM was established as the basis of the ALS metric and as a tool for technology selection. (Drysdale 1998) (Maxwell and Drysdale 2001) A detailed historical review of the definitions of equivalent mass and ESM is given in Appendix A.

ESM is simply the traditional equivalent mass with the addition of mass representing crew time and "other costs." We first describe traditional equivalent mass and then ESM.

EQUIVALENT MASS COMPUTATION - How do we compute traditional equivalent mass? Equivalent mass includes mass, $\mathbf{m}$, volume, $\mathbf{v}$, power, $\mathbf{p}$, cooling, $\mathbf{c}$, and logistics, I. More precisely, equivalent mass (EM) is equal to the sum of the system mass, $m$, the equivalent mass of the required volume, $E M(v)$, the equivalent mass of the required power supply, $E M(p)$, the equivalent mass of the required cooling system, EM(c), and the mass (M) of the materials and spares logistics, $M(I)$.

$$
\begin{aligned}
E M(\text { system })= & m+E M(v)+E M(p)+E M(c)+M(l) \\
& =E M(m, v, p, c, l)
\end{aligned}
$$

Equivalent mass is easily computed if we have the estimated mass and volume and the power, cooling, and logistics needs for a system. Traditional equivalent mass is based on these fundamental, easily estimated, physical quantities. Values of these parameters for some systems have been obtained from prototype data and have been published for some ALS components. (Eckart 1996) (Carrasquillo et al. 1992) 
Two of the quantities in the equivalent mass of a system are masses themselves. $m$ is the sum of the launch weights of all the equipment in the system. $M(I)$ is the sum of the launch weights of all the logistics (spares and resupplied materials) needed by the system for the duration considered.

Three other quantities are not masses. Volume, power, and cooling must be converted from cubic meters or Watts into kilograms. This is done using conversion factors called the mass-equivalents of volume, power, and cooling. For any required factor not measured in mass units, the equivalent mass, EM, is computed as the required quantity, $f$, of the factor times the massequivalent of the factor, me(f).

$E M(f)=f^{*} m e(f)$

How do we find the equivalent mass of the pressurized volume required by the system? Given a structure with a known mass that provides a certain pressurized volume, we divide the mass by the volume to determine the mass-equivalent of volume, me(v), as so many kilograms per cubic meter.

$\operatorname{me}(v)=[$ mass of structure $] /[$ volume of structure $]$

me(v) obviously is larger for steel than for inflatable modules. Multiplying the pressurized volume required by our system, $\mathbf{v}$, by the mass-equivalent of volume, me(v), we obtain the equivalent mass of the system's volume.

$E M(v)=v^{*} m e(v)$

The equivalent mass of the system's allocated portion of the power supply is found similarly. The power supply requires a certain launch mass and is at least partly housed in pressurized volume. Thus the power supply itself has an equivalent mass based on its own mass and required volume.

$E M($ power supply $)=m+E M(v)$

The power supply equivalent mass is divided by the power supply capacity in Watts to determine the massequivalent of power, me(p).

$m e(\mathbf{p})=E M($ power supply) $/$ [capacity of power supply]

$m e(p)$ is larger for solar than nuclear power. Multiplying the power required by the life support system, p, by the mass-equivalent of power, me(p), we obtain the equivalent mass of the system's allocated portion of the power supply.

$E M(p)=p^{*} m e(p)$

The equivalent mass of the system's allocated portion of the thermal cooling system is found similarly. The cooling system requires a certain launch mass and is partly housed in pressurized volume. The cooling system has an equivalent mass based on its mass and required volume.

$E M($ cooling $)=m+E M(v)$

The cooling system equivalent mass is divided the cooling system thermal capacity to determine the massequivalent of cooling, me(c).

$m e(c)=E M($ cooling $) /[$ cooling capacity $]$

Multiplying the cooling required by our system by the mass-equivalent of cooling, we obtain the equivalent mass of the system's cooling.

$E M(c)=c^{*} m e(c)$

Including mass, volume, power, cooling, and logistics, we have

$E M(m, v, p, c, I)=m+E M(v)+E M(p)+E M(c)+M(I)$

There are some minor ambiguities in the calculation of equivalent mass. The power supply requires cooling and the cooling system requires power. The pressurized volume itself requires active environmental control and uses power and cooling. Sometimes the equivalent mass of volume is neglected for the power supply, cooling system, and even for the system hardware itself. (See Appendix A.) Although the allocation of mass to different systems can be somewhat arbitrary, we can confirm at the launch vehicle level that all the launch mass is allocated reasonably, to one and only one subsystem.

\section{ESM (EQUIVALENT SYSTEM MASS)}

ESM was established by NASA Advanced Life Support for use in the ALS Metric (Drysdale and Hanford 1999). ESM has been used in NASA technology project selection (Maxwell and Drysdale 2001). ESM includes traditional equivalent mass described above (mass, power, volume, cooling, and logistics) with the addition of masses representing the crew time to operate the system and possibly "other cost factors."

Drysdale presented the definitive description of ESM at an ALS Systems Workshop on March 26, 1998. His chart "Equivalent System Mass - The Concept" reads:

"Equivalent mass is the total of all masses essential to a life support system including fixed and time-dependent masses of the system, mass of pressure vessels amortized for the life support system and any crew access required for the life support system (e.g. O\&M), amortized mass of power systems required to run the life support system, amortized mass of heat rejection for electrical power used and any process heat (e.g. combustion, metabolism), mass of life support for the 
- portion of crew time needed for systems operations and maintenance, (and) anything else that can be defined in terms of mass."

"Amortized" here means allocated or apportioned to the life support system. "O\&M" is operations and maintenance. (Drysdale 1998)

Drysdale presented a very similar definition in a teleconference on ESM on March 11, 2002.

"ESM $=m i+m v+m p+m c+m c t+m l+$ other cost items, where $\mathrm{mi}=$ initial mass, $m v=$ mass of pressurized volume, $\mathrm{mp}=$ mass of power required, $\mathrm{mc}=$ mass of cooling needed, mct $=$ mass for crew time required, and $\mathrm{ml}=$ logistics mass." (Drysdale 2002)

In both presentations four years apart, ESM includes traditional equivalent mass (mass, volume, power, cooling, and logistics) plus crew time and "other cost items" or "anything else that can be defined in terms of mass."

The papers describing ESM as differentiated from traditional equivalent mass) that are reviewed in Appendix A all include crew time but none mention "other cost items" or "anything else" as a factor. However, these papers all provide inclusive definitions of ESM. ESM includes "mass penalties for infrastructure support (notably power and cooling)," (Drysdale and Hanford 1999) or "the mass of all entities, including the structure required for pressurized volume, power system, and cooling system, that are required to make a life support system (LSS) function as intended." (Levri et al. 2000) The general terms "infrastructure" or "all entities ... required" would allow "other cost items" to be included.

Why include crew time and "other cost items"? Because "total cost must be considered" and "crew time is such a significant CELSS (Controlled Ecological Life Support System) cost factor." (Drysdale et al. 1992) Drysdale et al. wanted to consider total cost and to include all important costs. Unfortunately there are problems in computing the equivalent mass of crew time and identifying the "other cost items" in ESM. "Other cost items" has always been undefined. No examples were found in the literature reviewed in Appendix A. In the March 11, 2002, teleconference where the definition and calculation of ESM were described, "other cost items" was strongly objected to and declared inoperative.

The current ALS definition of ESM is traditional equivalent mass plus the equivalent mass of crew time. Unlike traditional equivalent mass, ESM does not correspond to a simple physical concept. The traditional equivalent mass of a system includes all of the launch mass required to fly and operate the system. The equivalent mass of the crew time required to operate a system is an additional penalty mass that may be based on the equivalent mass or some other mass. (Jones 2001) Even with the addition of crew time, ESM crew time falls short of representing total cost, since it does not reflect design, development, and operations costs. The justification for ESM is that launch cost is significant, crew time cost is significant, and these two costs are much larger than other costs. (Drysdale 1998) This is not always true.

How is the equivalent mass of crew time computed? Two methods are used in ALS. (BVAD 2002) (Levri et al. 2000) (Jones 2001) Appendix B describes these methods and shows they give the same result for small added amounts of crew time. The equivalent mass of crew time is a non-physical mass penalty that is added to traditional equivalent mass. The equivalent mass of crew time becomes infinitely large as the crew time required for maintenance and operations approaches the total crew time available.

The computation of the equivalent mass of crew time is based on the assumption that crew time is over subscribed, which may not be true in transit. It also assumes that the purpose of all crew time is to conduct science, which may not be true if the mission goal is building a base. If crew time is underutilized, it is not appropriate to assign any cost to crew time. (Jones 2001)

Because of the conceptual difficulties created by adding the equivalent mass of crew time and "other cost factors" to ESM, traditional equivalent mass is a simpler, clearer, and more useful metric than ESM. Traditional equivalent mass is the allocated launch mass and is a good indicator of launch cost. ESM does not represent a single, clear, identifiable cost.

\section{APPLICATION OF EQUIVALENT MASS}

Even when we merely want to know the launch mass needed to provide a particular system, there are two significant problems in using equivalent mass. These are the effects of location and of mass reduction on the actual mission cost.

LOCATION - We first consider the effect of system location. The justification for using equivalent mass as a cost metric is that launch cost is a large portion of the cost of a planetary mission and that launch cost is proportional to launch mass. This is reasonable, but most of the mass that is launched to low Earth orbit is not in the payload used on the planetary mission, but in the rockets and propellant that carry the payload to the planetary location. Of course, the mass needed for propulsion is proportional to the mass of the payload. The problem is that different life support systems and materials are used in different locations, in transit to the planet, orbit, descent, ascent, and transit back to Earth. (Fisher 2003) Because the propulsion system used in one travel stage is part of the payload for the previous stages, payload mass becomes several times more expensive with each 
successive stage. The cost of mass delivered to a planetary surface is many times higher than the cost of mass in low Earth orbit (LEO). And the cost of propulsion to the same location can differ greatly if different propulsion methods are used. Payloads can be sent in advance of humans using slow, low cost electric propulsion. Or rapid transit can be achieved at a higher cost.

Equal equivalent mass on the launch pad has a vastly different cost to the mission if different masses are going to different locations or to the same location using different propulsion methods. Equivalent mass is used as if all mass, volume, power, cooling, and logistics have the same cost to the mission, as if they were all going to the same location in the same way. This is extremely unrealistic for a complex planetary mission. Much of the mass that is launched will remain on the planetary surface and practically none will return to Earth. The equivalent mass of a system is the same whether we leave it on the planet or bring it back to Earth, but the emplacement cost is much different. (Fisher 2003)

MASS REDUCTION - There is another problem in using equivalent mass because equipment for planetary missions is usually redesigned to reduce mass. Suppose we frequently need to send a pump to a very remote location. The pump costs $\$ 1,000$ and weighs $10 \mathrm{~kg}$ off the shelf. Cost is $\$ 100$ per $\mathrm{kg}$. We find that the shipping for some reason costs $\$ 100,000$ per $\mathrm{kg}$. This is $\$ 1,000,000$ to ship one $10 \mathrm{~kg}$ pump. The final delivered cost is $\$ 100,100$ per $\mathrm{kg}$. It is cost-effective to spend several $\$ 10,000$ per $\mathrm{kg}$ to reduce the weight of the pump. Similar considerations apply to planetary missions. It is usual for robotic planetary missions to spend considerable development money to reduce equipment mass. The result is that, even though emplacement cost is very high, the development cost per kilogram can be even higher for planetary missions. In this example, suppose we can reduce the pump weight to $4 \mathrm{~kg}$. The shipping cost is then $\$ 400,000$ and we save money as long as the modified pump cost is less than $\$ 600,000$. The design and development cost can be larger than the delivery cost. The weight reduction effort can increase the cost of the pump per kilogram up to $\$ 600,000 / 4 \mathrm{~kg}=$ $\$ 150,000 / \mathrm{kg}$. This design and development cost per kilogram is significantly larger than the delivery cost per kilogram. Launch cost may not dominate mission cost even on a planetary mission, contrary to the fundamental assumption justifying the use of equivalent mass as a cost metric. Of course, life support materials such as oxygen, water, and food solids can not themselves be reduced in mass. The equivalent mass calculation treats individual subsystem mass as fixed and determinable during preliminary system design. It does not consider the usual intense redesign efforts to reduce mass.

ESM is the designated metric for reporting ALS progress and is often considered in ALS research funding and technology project approval. However, we need to beware of the problems described above.

It is best to use equivalent mass to compare the mass of systems to be sent to the same location by the same type of propulsion. All the mass must have the same propulsion cost. We can use equivalent mass to compare two planetary life support systems, or two transit designs, but should not use it to compare open loop systems that vent and dump waste with closed loop systems that carry all initial materials throughout a mission.

It is best to use equivalent mass to compare systems with similar potential for weight reduction. We can use equivalent mass to compare two prototype physicochemical recycling systems, but not to compare brass board recycling hardware with an open loop system that requires a large initial mass of stored material and continually discards waste. The latter system has a different potential for mass reduction and uses mass in different locations.

ORDER OF MAGNITUDE ACCURACY - "Another word of caution about the equivalent-mass concept - it provides only a rough order-of-magnitude calculation for comparing LSS configurations in the very early stages of mission planning." (Doll and Eckart 2000, p. 566)

The limited technical information available early in the project cycle and the approximations used in equivalent mass reduce its accuracy to within an order of magnitude.

Equivalent mass can provide a summary of a more complete analysis. Even when equivalent mass and only equivalent mass is presented to justify technology selection, it is rarely considered sufficient. Decision makers want to know the details, including the systems' mass, volume, power, cooling, logistics, and crew time requirements. They also want to know the power and volume mass equivalents and if solar or nuclear power and if a steel or inflatable structure was assumed.

It is probably better that traditional equivalent mass be used, including only mass, volume, power, cooling, and logistics, rather than ESM with its confusing addition of the equivalent mass of crew time. Crew time should be considered separately, since its importance depends on anticipated crew work load.

Equivalent mass is proportional to and represents launch cost. Equivalent mass should not be used exclusively for technology selection if other costs are equally or more important. International Space Station (ISS) cost $\$ 30-40$ billion for design, development, test and evaluation (DDT\&E) and only \$3-4 billion to launch. Launch cost is not included in cost summaries. Operations cost may equal DDT\&E cost. ISS technology selection choices were based on DDT\&E cost, not launch cost. 
The purpose of creating ESM by adding crew time and "other costs" to traditional equivalent mass was to include the most important costs. The benefit of ESM is that engineers and managers have a relatively straightforward method to include mass and cost considerations in design decisions. Using ESM is much better than ignoring the system-wide impact of mass and mass related costs. ALS should apply much more effort to mass reduction. The problem with ESM is that it does not correctly show the effect of mass, so that the actual mission cost impact will be much different than ESM indicates. ESM is not a good estimator for mission cost of even for launch cost.

It would be possible to create a more useful cost metric that included the development and operations cost and also reflected the actual emplacement cost rather than only the launch cost. However, it seems better to simply identify and combine all the mission costs, including DDT\&E, launch and emplacement, and operations. These are the major elements of the Life Cycle Cost (LCC) that will be considered below.

\section{DDT\&E (DESIGN, DEVELOPMENT, TEST, AND ENGINEERING) COST}

Parametric cost models provide the most effective way to estimate the DDT\&E cost of space hardware early in the mission cycle. Parametric cost models use cost estimating relationships (CERs) that appraise the cost of a system using its quantitative characteristics. The most common cost determining parameters are the system dry mass and the quantity to be produced.

NASA uses three different parametric models for crewed space systems; the commercial PRICE-H space hardware cost model, the NASA-Air Force Cost Model (NAFCOM), and the Advanced Missions Cost Model (AMCM).

PRICE - The PRICE parametric cost models are those most frequently used in aerospace. PRICE models exist for hardware development, hardware maintenance, software development, and scheduling. PRICE-H estimates the hardware development and production cost. There are different models for different mission types. The basic input parameters are two masses, the active electronics mass and the less costly mechanical/structural mass. The most important elements of the model are the costs per unit mass for electronics and mechanical/structure. These are obtained from tables or by calibrating the model using relevant past costs. The third input parameter is the engineering complexity, which reflects the scope of the development task, the skills of the development organization, and the amount of design repetition. The number of development and production units is required and a production learning curve may be defined. (Guerra and Shishko 2000, p. 945) (Greenberg 1992, p. 85)
NAFCOM - The NAFCOM model was developed by Marshall Space Flight Center. Each CER in the NAFCOM uses a single input parameter such as mass, power, or thrust, and gives estimated development and production cost in 1987 dollars. There are specific cost models for crewed spacecraft systems, including environmental control and life support. Systems engineering, testing, integration, and program management are estimated separately and added. Complexity factors are applied to the CERs. (Guerra and Shishko, pp. 945, 7) The NAFCOM software is available for download at no cost. (http://nafcom.saic.com/)

AMCM - The Exploration Programs Office at Johnson Space Center developed the AMCM (Advanced Missions Cost Model) to improve on models based only on mass. The AMCM is appropriate early in conceptual design where little detailed data is available. The model is a single CER using mass, quantity, mission type, number of design generations, and technical difficulty to estimate the total system cost for DDT\&E and production.

The AMCM formula for the cost of DDT\&E and production in millions of 1999 dollars is:

Cost $=10.59 Q^{0.59} M^{0.66} 80.6^{\top} G^{-0.36} 1.57^{D}$

$Q$ is the total quantity of development and production units, $M$ is the system dry mass in kilograms, $T$ calibrates for the type of mission (2.14 for human habitat, 2.4 for crewed planetary), $G$ is the hardware generation ( 1 for new design, 2 for second generation), and $D$ is the estimated difficulty ( 0 for average, 2 for very difficult, and -2 for very easy). (Guerra and Shishko, pp. 946-7) (Greenberg, pp. 77-84)

The mission type will be the same for all the life support system technologies being compared, but the other parameters may differ. If the quantity is one, the mission is crewed planetary, the design is first generation, and the difficulty is average, the formula reduces to Cost = $10.59 \mathrm{M}^{0.66}$. For equipment weighing less than $1,000 \mathrm{~kg}$, the estimated cost per kilogram is more than one million dollars. This is much higher than for the Shuttle or ISS, which are not planetary missions. For two units, $Q=2$, the total cost increases only by $50 \%$. The cost for a second generation design is 78 percent of that for a first generation design. A very difficult development with $D=$ 2.5 increases estimated cost three times, while a very easy development with $D=-2.5$ decreases cost to onethird. A significant cost adjustment can be made by considering difficulty of the current development compared to past programs of the same type. (The JSC AMCM is online at

http://www.jsc.nasa.gov/bu2/AMCM.html)

For equipment for a human space habitat $(T=2.14)$, with quantity of 4 , mass of $100 \mathrm{~kg}$, a second generation design, and very low difficulty $(D=-2.5)$, the total cost is roughly $\$ 40$ million. The cost per delivered kilogram for 
four $100 \mathrm{~kg}$ units is $\$ 40,000 \mathrm{k} / 400 \mathrm{~kg}=\$ 100 \mathrm{k} / \mathrm{kg}$. This is similar to the Shuttle or ISS costs per $\mathrm{kg}$. The DDT\&E cost for human missions is typically $\$ 100 \mathrm{k} / \mathrm{kg}$, with a range of from $\$ 50$ to $150 \mathrm{k} / \mathrm{kg}$. (Wertz and Larson 1996, p. 254) (National Academy Press, App. C) (Larson and Pranke 2000, p. 953)

The AMCM model uses familiar input parameters. Cost decreases with the quantity of units, $Q$, due to the effect of experience reflected in the learning curve. $Q$ includes development units, credit for partial units and mock-ups, test units, and flight units. The dry mass, $M$, is the most frequently used cost estimation parameter. Different types of missions have had very different costs per kilogram. The type of mission, $T$, is designed to allow the AMCM formula to include a range of missions. Cost decreases with the level of design inheritance. This is reflected in the number of modifications or past generations, $G$.

The most subjective parameter in AMCM is the difficulty, D. Adjustments for difficulty or complexity are also used in the PRICE and NAFCOM models. The difficulty parameter adjusts for differences in technology, materials, manufacturing processes, parts count, design inheritance, schedule, the number of different organizations involved, and even the developing organization's experience and culture. (Guerra and Shishko, p. 947)

The total DDT\&E cost and the cost per kilogram depend strongly on the type of mission. Costs are much higher for planetary missions than for Earth orbit missions. This is partly due to the need for higher performance and reliability, but more because of the much higher emplacement cost per kilogram for planetary missions. Reducing system mass as much as possible for missions beyond LEO significantly increases the total DDT\&E cost and the cost per kilogram while reducing the mass, emplacement cost, and total mission cost. We next consider launch and emplacement cost.

\section{LAUNCH AND EMPLACEMENT COST}

The Space Shuttle cost to launch to LEO is typically quoted as $\$ 25 \mathrm{k} / \mathrm{kg}$. (Wertz and Larson 1996, p. 125) A yearly Space Shuttle budget of 4 billion dollars for 10 launches of $16,000 \mathrm{~kg}$ to LEO corresponds to a cost of \$ $25 \mathrm{k} / \mathrm{kg}$.

For a Moon or Mars mission, we must launch to LEO the payload and the propulsion system - the vehicle and propellant - needed to get the payload to the planetary surface or back to LEO. A rough estimate of the propulsion cost uses the ratio of the initial mass to the final mass after the required mission maneuvers. The initial mass includes the propellant but the final mass does not. These initial/final mass ratios depend on the required $\Delta-v$ and the specific impulse of the propellant. Typical initial/final mass ratios from LEO to a Moon or Mars landing are shown in Table 1 below. (Larson and Pranke 2000, p. 276)

Table 1. Initial/final mass ratios from LEO to Moon or Mars.

\begin{tabular}{|l|l|l|}
\hline & Moon & Mars \\
\hline $\begin{array}{l}\text { LEO to Moon or Mars } \\
\text { orbit }\end{array}$ & 3.7 & 3.8 \\
\hline Deorbit and landing & 1.8 & 1.3 \\
\hline Combined (product) & 6.7 & 4.8 \\
\hline
\end{tabular}

The table shows the mass ratios for missions leaving LEO, orbiting the Moon or Mars, then deorbiting and landing. The Moon mission requires rocket powered lunar orbit insertion and descent. The Mars mission uses aerobraking for Mars orbit insertion followed by a two phase - parachute and powered - descent. The combined product mass increase ratios indicate that we need to launch to LEO propellant that is 4.8 to 6.7 times as massive as the planetary surface payload. The mass of the vehicles is ignored here but included below after table 2.)

The initial/final mass ratios from LEO to Moon or Mars orbit and a return to LEO are shown in table 2 below. (Larson and Pranke 2000, p. 276)

Table 2. Initial/final mass ratios from LEO to Moon or Mars orbit and back to LEO.

\begin{tabular}{|l|l|l|}
\hline & Moon & Mars \\
\hline $\begin{array}{l}\text { LEO to Moon or Mars } \\
\text { orbit }\end{array}$ & 3.7 & 3.8 \\
\hline $\begin{array}{l}\text { Moon or Mars orbit to } \\
\text { LEO }\end{array}$ & 1.4 & 3.5 \\
\hline Combined (product) & 5.0 & 13.2 \\
\hline
\end{tabular}

The first row is the same as table 1 . The combined mass increase ratios indicate that we need to boost 5.0 to 13.2 times the mass of the returning life support system to LEO initially.

Tables 1 and 2 indicate the launch mass for planetary missions can be roughly 5 to 13 times the payload launch mass. Based on Space Shuttle launch costs to LEO of $\$$ $25 \mathrm{k} / \mathrm{kg}$, the planetary emplacement or LEO return costs are $\$ 125$ to $325 \mathrm{k} / \mathrm{kg}$. 
The launch cost is significantly higher than this since the cost of launching the transit and landing vehicles must be added. Tables 1 and 2 above give the initial to final mass ratios for each stage of travel. The initial mass includes the payload, the propellant, and the vehicles. The final mass includes the payload and the final vehicle. The above cost estimates assumed all the vehicles had zero mass. It is more correct to estimate the vehicle mass as $15 \%$ of the propellant mass. (Larson and Pranke 2000 , p. 405) (Wertz and Larson 1996, p. 142) We must launch vehicles weighing $15 \%$ of the propellant mass, so that the cost of launch to LEO is higher than estimated above.

The calculation of the combined (product) initial/payload mass ratio in LEO considers the final payload landed or returned to LEO, the second stage landing or return rocket and propellant, and the first stage LEO to Moon or Mars orbit rocket and propellant. This calculation is given in Appendix $C$. The mass ratios are significantly higher than in tables 1 and 2. For a Moon landing or return to LEO, and for a Mars landing, the initial/payload mass ratio is roughly 20 . For Mars orbit and return to LEO, the initial/payload mass ratio is roughly 50 .

Suppose that the total initial mass of the life support system, the propellant, and the vehicles is 20 times the mass of the life support system alone. Table 3 below shows the initial masses at LEO for an initial/payload mass ratio of 20 .

Table 3. Payload, vehicle, and propellant masses at LEO.

\begin{tabular}{|l|l|}
\hline Payload mass & 1.0 \\
\hline Second stage vehicle mass & 0.3 \\
\hline Second stage propellant mass & 1.2 \\
\hline First stage vehicle mass & 2.4 \\
\hline First stage propellant mass & 15.1 \\
\hline Total initial mass & 20.0 \\
\hline
\end{tabular}

The vehicle mass is $15 \%$ of the propellant mass. The vehicle and propellant mass is $95 \%$ of the initial mass and the life support system payload is $5 \%$

Table 4 below shows the estimated cost of a kilogram emplaced on the Moon or Mars surface or brought back from Moon orbit to LEO, assuming the payload, vehicle, and propellant masses in Table 3.
Table 4. Payload, vehicle, propellant, and launch costs for a planetary mission.

\begin{tabular}{|l|r|r|r|}
\hline & $\begin{array}{l}\text { lost } \\
\text { factor }\end{array}$ & $\begin{array}{l}\text { mass } \\
\text { ratio }\end{array}$ & $\begin{array}{l}\text { \$k/kg } \\
\text { payload }\end{array}$ \\
\hline payload & $500 \mathrm{k} / \mathrm{kg}$ & 1 & 500.0 \\
\hline vehicles & $5 \mathrm{k} / \mathrm{kg}$ & 2.7 & 13.5 \\
\hline propellants & $0 \mathrm{k} / \mathrm{kg}$ & 16.3 & 0.0 \\
\hline cost to launch & $25 \mathrm{k} / \mathrm{kg}$ & & 500.0 \\
\hline cost at LEO & & & $1,013.5$ \\
\hline
\end{tabular}

The cost of $\$ 500 \mathrm{k} / \mathrm{kg}$ for a planetary payload is typical and may be an underestimate. The cost of rocket vehicles is low, $\$ 5 \mathrm{k} / \mathrm{kg}$, and the cost of propellant is negligible. (Wertz and Larson 1996, p. 126) The launch cost is equal to the payload DDT\&E cost, not much larger as often assumed. The launch and emplacement cost of $\$ 500 \mathrm{k} / \mathrm{kg}$ is not unexpected and may even be low. The launch and emplacement cost of a kilogram sent to Mars orbit and returned to LEO is significantly larger, $\$ 25 \mathrm{k} / \mathrm{kg}$ times the initial/payload mass ratio of 50 , or $\$ 1,250 \mathrm{k} / \mathrm{kg}$. Such costs are not unexpected. "The cost of a humancrewed mission to the Moon or Mars is typically millions of dollars per delivered kg." (Wertz and Larson 1996, p. 254)

\section{OPERATIONS COST}

The operations phase of a human space mission begins when Earth orbit is achieved and continues until the crew is returned safely to Earth. The payload design complexity and the mission duration are the two major drivers of operations cost.

The operations cost for life support includes the material and spares cost, spacecraft crew time cost, ground control crew cost, and sustaining engineering. Long term bases require resupply, but limited duration visits probably will not. The resupply cost depends on its material cost, usually low for air, water, and food but high for spare parts, and the launch cost which is high for LEO and much higher for emplacement beyond LEO. The time the crew must spend on operations and maintenance can be a significant distraction from other work, but the appropriate cost depends on the total crew work loading. The ground crew cost depends on the size of the ground crew and the duration of the mission. Human missions usually require four or five teams for 24 hour, 7 day coverage. Sustaining engineering provides on-call expertise for maintenance and trouble shooting. Operations costs can be reduced by decreasing resupply, simplifying design, using efficient operations concepts, increasing resource margins, designing 
simple human interfaces, providing autonomy on board, and automating ground control.

The cost of resupply depends largely on the cost of launch and transportation and should be included in that category. The cost of space crew time presents special issues discussed in Appendix $B$ and elsewhere. We consider here only the cost of the ground operations to sustain the life support system.

Space operations costs can be approximately predicted as a percentage of the system development cost. Mission constraints and systems design choices tend to drive DDT\&E and operations costs in the same direction. Complex human missions, severe environments, and higher risk aversion increase both DDT\&E and operations cost. New technology, large amounts of software, complex monitoring and control, tight margins, and multiple operational modes do also. (Wertz and Larson 1999, p. 612-4)

Yearly operations cost is usually estimated as some percentage of the DDT\&E cost. Human space missions before Shuttle usually spent a relatively small amount of the total budget on operations because the amount of time in space was relatively brief. The Shuttle's total cost including ten years of operations is $58 \%$ operations, and the operations cost is $157 \%$ of the DDT\&E cost. Shuttle operations cost roughly $16 \%$ of DDT\&E per year, but this includes launch, which we count separately. The estimated total cost of the ISS including ten years of operations is $51 \%$ operations, and the total operations cost is $113 \%$ of the DDT\&E cost. ISS operations cost roughly $11 \%$ of DDT\&E per year, not including launch. (Guerra and Shishko 2000, p. 938)

The Johnson Space Center (JSC) Mission Operations Cost Model (MOCM) estimates the operations cost as a percentage of the total DDT\&E and production cost of the spacecraft. For manned spacecraft, the operations cost per year is $10.9 \%$ of the total DDT\&E and production cost. The model is based on spacecraft data before 1990 and includes other types of mission. The operations cost includes ground systems, mission control and planning, data analysis, and crew training. It does not include launch. (The JSC MOCM is online at http://www.jsc.nasa.gov/bu2/MOCM.html)

\section{LIFE CYCLE COST (LCC)}

One major objective of ALS research is to minimize the total life cycle cost (LCC) of mission life support. LCC is a basic metric in systems analysis and is usually part of NASA mission design. LCC includes the costs incurred for life support during the development, launch and emplacement, and operations phases of a human space mission. The relative cost of these mission phases varies and some costs can be traded between mission phases, so we should design life support to minimize the total mission cost rather than the cost of any one phase.
The proportions of DDT\&E, launch and emplacement, and operations cost in the total mission cost depend on the mission location and duration. For manned space missions to LEO budgeted for ten years, such as Shuttle and ISS, the mission cost breakdown is typically $5 \%$ for analysis and definition (phases A/B), 40\% for DDT\&E (phase C/D), $15 \%$ for launch (phase E), and $40 \%$ for operations (phase F). (Guerra and Shishko 2000, p. 938) The life support budget for future LEO missions could have a similar breakdown, so that DDT\&E and operations costs would be much more important than launch costs.

For a five year mission beyond LEO such as to the Moon or Mars, the total mission cost would be higher. Following Table 4 above, we can assume a DDT\&E cost of $\$ 500$ $\mathrm{k} / \mathrm{kg}$ for a planetary payload, and a launch and emplacement cost of $\$ 513.5 \mathrm{k} / \mathrm{kg}$ including the vehicles. The operations cost is roughly $11 \%$ of DDT\&E per year, $\$ 55 \mathrm{k} / \mathrm{kg}$ per year, and $\$ 275 \mathrm{k} / \mathrm{kg}$ for five years. Analysis and definition is assumed to be $5 \%$ of the total. These amounts are shown in Table 5 below.

Table 5. Mission phase costs for a planetary mission.

\begin{tabular}{|c|c|r|r|}
\hline Phase & \multicolumn{1}{|c|}{ activity } & $\begin{array}{c}\text { \$k/kg } \\
\text { payload }\end{array}$ & percent \\
\hline A\&B & $\begin{array}{c}\text { analysis and } \\
\text { definition }\end{array}$ & 67.8 & 5 \\
\hline C/D & DDT\&E & 500.0 & 37 \\
\hline $\mathrm{E}$ & $\begin{array}{c}\text { launch and } \\
\text { emplacement }\end{array}$ & 513.5 & 38 \\
\hline $\mathrm{F}$ & 5 year operations & 275.0 & 20 \\
\hline & total & $1,356.3$ & 100 \\
\hline
\end{tabular}

The percentages of cost in each mission phase are also shown in Table 5. The budget breakdown is $5 \%$ for analysis and definition, $37 \%$ for DDT\&E, $38 \%$ for launch and emplacement, and $20 \%$ for operations. The change from the ten year LEO missions considered above is that the operations cost percentage is half and the launch cost is 2.5 times, in percentage terms.

The launch and emplacement cost for missions beyond LEO is not most of the total cost and it could be lower. The large launch cost to send payload mass beyond LEO requires and justifies a high investment in DDT\&E to reduce launch mass. This is usual in the design of robot planetary probes. The launch cost should not dominate total cost in a good mission plan. The correct approach for missions beyond LEO is to spend additional DDT\&E funds to decrease the mass and launch cost, in order to minimize the total mission cost. A mission that remains in Earth orbit has much less 
incentive to reduce mass than a mission going beyond LEO. ALS technology selection, mission design, and mass reduction efforts should use LCC to make the most effective decisions.

\section{CONCLUSION}

ISS did not use equivalent mass to select its life support technology. (Carrasquillo et al. 1992, pp. 2, 11) ISS technology selection placed more reliance on Technology Readiness Level (TRL). There is a very good reason for this. ISS is not going beyond LEO. It does not require vehicles and propulsion fuel to be launched to LEO for travel beyond LEO. Transporting hardware to LEO requires only $\$ 25 \mathrm{k}$ per payload kilogram, and not the additional $\$ 500-1,250$ per payload kilogram that it costs for the fuel to place the payload on the Moon or Mars or return it to LEO. The cost for space hardware Design, Development, Test, and Evaluation (DDT\&E) for LEO is typically $\$ 50-150 \mathrm{k} / \mathrm{kg}$. This DDT\&E cost per kilogram is several times larger than the $\$ 25 \mathrm{k} / \mathrm{kg}$ for a Space Shuttle launch to LEO. Higher TRL usually indicates lower DDT\&E cost. ISS selected technology with high TRL to minimize its high DDT\&E cost. ISS did not try to minimize its smaller launch cost by selecting technology with low equivalent mass

Should ALS use equivalent mass or Life Cycle Cost in technology selection? Each should be used as appropriate, but LCC has some advantages. Neither is much simpler or easier to compute than the other. Both have a clear central concept and both have extensive unavoidable complications. Neither is more accurate than the other. Both provide only a rough order of magnitude estimate intended for preliminary analysis. Equivalent mass in the form of ESM has been prescribed for use in ALS, while LCC is used widely in NASA, aerospace, and industry. Equivalent mass has a limited literature and experience base, while LCC has much more.

The main reason to use LCC in preliminary technology selection is that it considers the total mission cost. Equivalent mass is a proxy for launch cost alone, which excludes most of the mission cost. Development, operations, and even emplacement costs are based on other cost factors in addition to mass. Defining the estimated LCC begins the process of cost tracking and control and helps to reduce the real cost. LCC can incorporate NASA cost estimation research for DDT\&E, launch, and operations. Using LCC will increase awareness of the high cost of human space missions and encourage efforts to reduce cost. This contrasts with equivalent mass, which was devised partly to avoid emphasizing the problem of high costs.

As we consider using equivalent mass or LCC as cost metrics, we must remember that cost is not everything. Many other factors affect mission design and technology selection. These include system performance, risk, reliability, and safety.

\section{ACKNOWLEDGMENTS}

John Fisher first showed that the equivalent mass of a system depends on the mass locations. Alan Drysdale, Mike Ewert, John Fisher, Anthony Hanford, and Julie Levri provided valuable discussions. The reviewers are thanked for their encouragement, corrections, and detailed suggestions.

\section{REFERENCES}

1. BVAD, Advanced Life Support Baseline Values and Assumptions Document, JSC CTSD-ADV-484, 5/8/2002.

2. Carrasquillo, R. L., D. L. Carter, D. W. Holder, Jr., C. F. McGriff, and K. Y. Ogle, Space Station Freedom Environmental Control and Life Support System Regenerative Subsystem Selection, NASA TM4340, February, 1992.

3. Doll, S., and P. Eckart, "Environmental Control and Life Support Systems (ECLSS)," in W. K. Larson, and L. K. Pranke, eds., Human Spaceflight: Mission Analysis and Design, McGraw-Hill, New York, undated, 2000.

4. Drysdale, A., "Metrics and System Analysis," SAE Technical Paper No. 981746, Society of Automotive Engineers, Warrendale, PA, 28th International Conference on Environmental Systems, 1998.

5. Drysdale, Alan, Mark Thomas, Mark Fresa, and Ray Wheeler, "OCAM - A CELSS Modeling Tool: Description and Results," SAE Technical Paper No. 921241, Society of Automotive Engineers, Warrendale, PA, 22nd International Conference on Environmental Systems, 1992.

6. Drysdale, "Equivalent Mass and Infrastructure Costs," presented at the ALS Systems Workshop, March 26, 1998.

7. Drysdale, "ESM Calculation - current SIMA methods," teleconference presentation, March 11, 2002.

8. Drysdale, A.E., and Hanford, A.J., Advanced Life Support Research and Technology Development Metric - Baseline, CTSD-AD-384, JSC 39503, November, 1999.

9. Eckart, P., Spaceflight Life Support and Biospherics, Space Technology Library, Kluwer Academic, Dordrecht, 1996.

10. Fisher, J. W. "The Effect of Mission Location on Mission Costs and Equivalent System Mass," SAE Technical Paper No. 2003-01-2633, Proceedings of the 33rd International Conference on Environmental Systems, 2003.

11. Greenberg, J.S., "Appendix: CERs and Models" to "Cost-Estimating Relationships in Space Programs," in Space Economics, J.S. Greenberg and H.R. Hertzfeld, American Institute of Aeronautics and Astronautics, Washington, 1992.

12. Guerra, L., and R. Shishko, "Estimating the Cost of Crewed Space Systems," in W. J. Larson, and L. K. 
Pranke, eds., Human Spaceflight: Mission Analysis and Design, McGraw-Hill, New York, undated, 2000.

13. Jones, H. W., "The Cost and Equivalent System Mass of Space Crew Time," SAE Paper No. 2001. 01-2359, 31st International Conference on Environmental Systems, 2001.

14. Jones, H. W., "Multiple Metrics for Advanced Life Support," SAE Technical Paper No. 1999-01-2079, Proceedings of the 29th International Conference on Environmental Systems, 1999.

15. Jones, H. W., R. Dillon-Merrill, T.O. Tri, and D.L. Henninger," Reducing the Risk of Human Space Missions with INTEGRITY," SAE Technical Paper No. 2003-01-2572, Proceedings of the 33rd International Conference on Environmental Systems, 2003.

16. Larson, W. J, and W. Balogh, "Designing Human Space Missions," in W. J. Larson, and L. K. Pranke, eds., Human Spaceflight: Mission Analysis and Design, McGraw-Hill, New York, undated, 2000.

17. Larson, W. J., and L. K. Pranke, eds., Human Spaceflight: Mission Analysis and Design, McGrawHill, New York, undated, 2000.

18. Levri, J. A., D. A. Vaccari, and A. E. Drysdale, "Theory and Application of the Equivalent System Mass Metric," SAE Paper No. 2000-01-2395, 30 th International Conference on Environmental Systems, 2000.

19. Maxwell, S., and Drysdale, A., "Assessment of Waste Processing Technologies for 3 Missions," SAE Paper No. 2001-01-2365, 31st International Conference on Environmental Systems, 2001.

20. National Academy Press, Reducing the Costs of Space Science Research Missions, http://www.nationalacademies.org/ssb/jctmenu.html Washington, 1997.

21. Wertz, J. R., and W. J. Larson, eds., Reducing Space Mission Cost, Space Technology Series, Kluwer, Dordrecht, 1996.

22. Wertz, J. R., and W. J. Larson, eds., Space Mission Analysis and Design, third edition, Space Technology Series, Kluwer, Dordrecht, 1999.

\section{CONTACT}

Harry Jones, Ph.D.

Mail Stop 239-8

NASA Ames Research Center

Moffett Field, CA 94035-1000

Phone: 650-604-5518

e-mail: Harry.W.Jones@nasa.gov

\section{DEFINITIONS, ACRONYMS, ABBREVIATIONS}
ALS: Advanced Life Support
AMCM: Advanced Missions Cost Model
CER: Cost Estimating Relationship
DDT\&E: Design, Development, Test, and Evaluation
EM: Equivalent Mass
ESM: Equivalent System Mass
ISS: International space Station
LCC: Life Cycle Cost
LEO: Low Earth Orbit
LSS: Life Support Systems
M: Mass
me: mass-equivalent
NAFCOM: NASA-Air Force Cost Model
TRL: Technology Readiness Level 


\section{APPENDIX A: DEFINITIONS OF EQUIVALENT MASS AND ESM}

The concept of equivalent mass has been used in planning long duration human space missions since the Apollo era. Equivalent mass was originally called equivalent weight. Sometimes launch cost was computed using a total launch weight similar to equivalent mass. In 1998 NASA's Advanced Life Support Program established the use of a related concept, Equivalent System Mass (ESM), for Headquarters reporting and research project selection.

The purpose of the annotated bibliography and discussion below is to trace the definition of equivalent mass and ESM over time. It shows that equivalent mass has a traditional and well-understood meaning. The equivalent mass of a system includes the masses of the hardware itself, its spares and resupplied materials, and the allocated masses of the pressurized volume, power supply, and cooling needed to support the system. The equivalent masses of volume, power, and cooling are computed using mass equivalencies derived from the masses of the pressurized structure, power supply, and cooling system. Drysdale's concept of equivalent mass added a mass equivalent of crew time. With his participation, NASA ALS defined ESM to include crew time. Computing the equivalent mass of crew time is described in Appendix B.

ANNOTATED BIBLIOGRAPHY - The references are given in chronological order, earliest first.

[1970 ALISS] United Aircraft, Trade-Off study and Conceptual Designs of Regenerative Advanced Integrated Life Support Systems (ALISS), NASA CR1458, January 1970.

Equivalent mass was called equivalent weight on page 18 of this 1970 study. "Equivalent weight - Most physical aspects of any concept can be converted to an equivalent weight penalty for purposes of comparison. This tool is used in this study to provide an objective basis for evaluating such considerations as fixed weight, expendables, power and heat rejection requirements, spares and redundancy to achieve reliability goals, suppression devices to maintain noise levels, and control devices to prevent contamination. These factors are converted to and included in the equivalent weight of the concept."

Even at this early date, equivalent weight included mass, logistics, power, and cooling. Except for the volume penalty corresponding to the mass of the required structure, the equivalent weight used in 1970 had all the factors usually found in equivalent mass.

[1986 CELSS] Boeing Aerospace, Controlled Ecological Life Support System (CELSS) Physiochemical Waste Management Systems Evaluation, March 1986.
This 1986 study used the term launch cost, which was based on "on-orbit weight and volume, logistics weight and volume, power consumption, and heat rejection." But "(i)n relation to the total Shuttle capacity (on-orbit and logistics volume were) considered to be too small to justify comparison." "The launch costs for the subsystem on-orbit and logistics weight and the launch costs for the prorated power and thermal systems weight penalties can be totaled for each subsystem and compared." (pp. 52-4) Weights were converted to launch cost using the Shuttle capacity and estimated launch cost. Again mass, logistics, power, and cooling were included in launch cost while volume was considered but not used.

[1992 TRIALSS] Susan Doll and Brian Tillotson, "TRIALSS - Tool for Rapid and Intelligent Advanced life Support System Selection and Sizing," SAE Technical Paper No. 921123, Society of Automotive Engineers, Warrendale, PA, 22nd International Conference on Environmental Systems, 1992.

The terms "equivalent mass" "system equivalent mass" are well explained. 'A more objective 'apples to apples' measure is needed to perform mission level analysis. We have developed a measure called 'equivalent mass' which quantifies the life support system impact on power, volume, and heat rejection requirements. Mass penalties are assigned for the incremental masses of the extra power system needed to power the equipment, the pressure shell needed to enclose the hardware's volume and to store consumables and expendables for one resupply cycle, and the heat rejection system to dispose of waste heat from the equipment. Mass penalties for the power system, heat rejection system, and pressurized volume are technology dependent and are designated in $\mathrm{kg} / \mathrm{kWe}, \mathrm{kg} / \mathrm{kWt}$ and $\mathrm{kg} / \mathrm{m}^{3}$, respectively. The mass penalties for a given life support system are added to the mass of the life support system hardware, consumables, and expendables to generate the system equivalent mass." Equivalent mass as defined here includes mass, volume, power, cooling and logistics.

[1992 OCAM] Alan Drysdale, Mark Thomas, Mark Fresa, and Ray Wheeler, "OCAM - A CELSS Modeling Tool: Description and Results," SAE Technical Paper No. 921241, Society of Automotive Engineers, Warrendale, $P A$, 22nd International Conference on Environmental Systems, 1992.

The term "equivalent mass" is defined differently in the same conference as the previous paper. Manpower first appears here. "In evaluating mission options, total cost must be considered, including hardware design, development and test, hardware delivery, consumables and spares delivery, energy and manpower for operation and maintenance." (p. 1) "The four major cost drivers for an LEO manned space mission are design and fabrication, mass, energy, and manpower. Design and fabrication costs are disregarded in this analysis since: (a) 
most of the mass of a CELSS is module mass and low cost consumables mass, (b) fabrication of additional common modules is relatively low cost, and (c) module fabrication costs are much less than delivery costs. " ( $p$. 4) "Overall resource cost was calculated by taking the system mass and adding the equivalent masses of energy and manpower. This provides a single cost factor for evaluating system options. The lower the equivalent mass, the better the system." (p. 6) Equivalent mass here includes mass, power, and manpower.

[1995 Cost] Alan E. Drysdale, "The Effect of Resource Cost on Selection of Life Support Technologies," SAE Technical Paper No. 951492, Society of Automotive Engineers, Warrendale, PA, 25th International Conference on Environmental Systems, 1995.

"Costs include mass, pressurized volume, energy, cooling and manpower. They are converted to common mass units (equivalent mass)." "R\&D and fabrication costs are hard to estimate and are not considered."

[1995 LiSSA] Joe Ferrall, Gani B. Ganapathi, Naresh Rohatgi, and P. K. Seshan, "Parametric Studies Using LiSSA for an Extra-Terrestrial Manned Outpost," SAE Technical Paper No. 951495, Society of Automotive Engineers, Warrendale, PA, 25th International Conference on Environmental Systems, 1995.

Equivalent mass includes only "life support system weight" plus the mass equivalent of the power used.

[1997 OCAM 2] Alan Drysdale, "OCAM - 2: A Second Generation Bioregenerative Life Support System Model," SAE Technical Paper No. 972291, Society of Automotive Engineers, Warrendale, PA, 27th International Conference on Environmental Systems, 1997.

"To avoid subjective weightings, we have converted cost factors to mass units. The main cost factors for long duration space missions are thought to be equipment and consumables mass (measured in $\mathrm{kg}$ ), pressurized volume (m3), energy ( $k W h$ ), heat rejection (MJ), and crew time (man-hours). The required quantity of each cost factor needed for a particular mission scenario was estimated for the mission, converted into mass units using the appropriate equivalency, and the equivalent masses (EM) for the various cost factors were summed to provide an EM for that mission. The best mission scenario is the one with the lowest EM when other factors such as safety and reliability are equalized by design."

[1998 Metrics] Alan Drysdale, "Metrics and System Analysis," SAE Technical Paper No. 981746, Society of Automotive Engineers, Warrendale, PA, 28th International Conference on Environmental Systems, 1998.
"The metric I have used most to date has been equivalent system mass (ESM)." "ESM is the mass of all the resources needed to make the system work. From previous studies, the procurement cost of the equipment can as a first approximation be ignored for space systems, as it is outweighed by the delivery cost. The cost factors that have been generally used are: system mass $(\mathrm{kg})$, pressurized volume $(\mathrm{m} 3)$, energy, power (MJ, kW), heat rejection (MJ/day, $k W$ ), and crew time (whe)." Equivalent system mass (ESM) is the 1998 name for the exact same metric that was called equivalent mass in 1997 (OCAM 2).

[1999 ALS Metric] A.E. Drysdale and A.J. Hanford, Advanced Life Support Research and Technology Development Metric - Baseline, CTSD-AD-384, JSC 39503, November, 1999.

This is NASA's formal definition of the ALS metric, which is the ratio of the ESM's for ISS and ALS technology. ESM includes mass, volume, power, cooling, crew time, and logistics.

[1999 ESM] Alan E. Drysdale, Mike Ewert, Anthony J. Hanford, "Equivalent System Mass Studies of Missions and Concepts," SAE Paper No. 1999-01-2081, 29"th International Conference on Environmental Systems, 1999.

"Equivalent system mass (ESM) is used as a measure of goodness of life support systems. It is an estimate of the life support system mass required to perform a mission, including actual mass of equipment, system commodities, and resupply requirements (consumables, expendables, and spares); the mass of the pressure vessel to contain the LS system (including space for crew access); the amortized mass of the power and heat rejection infrastructure to support the LS system; and a mass number for the crew time required to operate and maintain the LS system."

[2000 ESM] Julie A. Levri, David A. Vaccari, and Alan E. Drysdale, "Theory and Application of the Equivalent System Mass Metric," SAE Paper No. 2000-01-2395, $30^{\text {th }}$ International Conference on Environmental Systems, July 10-13, 2000.

"The five components of ESM are mass, volume, power, cooling and crew time." Logistics was not mentioned but can be understood to be included. Reference is made to Drysdale and Hanford's 1999 ALS Metric baseline CTSDADV JSC 39503. The purpose of this paper is to explain the application of ESM. A method for computing the equivalent mass of crew time is developed.

[2000 ECLSS] S. Doll and P. Eckart, "Environmental Control and Life Support Systems (ECLSS)," pp. 565-6, in W. K. Larson, and L. K. Pranke, eds., Human Spaceflight: Mission Analysis and Design, McGraw-Hill, New York, undated, 2000. 
Doll and Eckart compute the required mass, volume, power consumption, thermal heat load, and crew time for operation and maintenance. They mention consumables, expendables, and spares. "The concept of equivalent mass established mass as a common unit." (pp. 564-5 ) Their equivalent mass includes mass, consumables, volume, power, and cooling, but not crew time. "The equivalent-mass approach does not account for important characteristics, such as reliability and maintainability in terms of spares and crew time requirements. Depending on the mission scenario, crew time may be one of the most important issues to evaluate." (p. 566)

DISCUSSION OF HISTORICAL EQUIVALENT MASS AND ESM - The occurrence of the different factors in equivalent mass and ESM is shown in table A-1 below. Mass (m), volume (v), power (p), cooling (c), logistics (I), and crew time (ct) are the only explicit factors that are ever included in equivalent mass. Internal NASA presentations of ESM have included "and other costs," but no specific costs were defined.

Table A-1. What is included in equivalent mass and ESM?

\begin{tabular}{|l|l|l|l|l|l|l|l|l|l|}
\hline & year & reference & author & m & v & p & c & l & ct \\
\hline 1 & 1970 & ALISS & United Aircraft & $x$ & & $x$ & $x$ & $x$ & \\
\hline 2 & 1986 & CELSS & Boeing & $x$ & & $x$ & $x$ & $x$ & \\
\hline 3 & 1992 & TRIALSS & Doll and Tillotson & $x$ & $x$ & $x$ & $x$ & $x$ & \\
\hline 4 & 1992 & OCAM & Drysdale et al. & $x$ & & $x$ & & & $x$ \\
\hline 5 & 1995 & cost & Drysdale & $x$ & $x$ & $x$ & $x$ & & $x$ \\
\hline 6 & 1995 & LiSSA & Ferrall et al. & $x$ & & $x$ & & & \\
\hline 7 & 1997 & OCAM 2 & Drysdale & $x$ & $x$ & $x$ & $x$ & $x$ & $x$ \\
\hline 8 & 1998 & Metrics & Drysdale & $x$ & $x$ & $x$ & $x$ & & $x$ \\
\hline 9 & 1999 & ALS Metric & Drysdale and Hanford & $x$ & $x$ & $x$ & $x$ & $x$ & $x$ \\
\hline 10 & 1999 & ESM & Drysdale, Ewert, Hanford & $x$ & $x$ & $x$ & $x$ & $x$ & $x$ \\
\hline 11 & 2000 & ESM & Levri, Vaccari, Drysdale & $x$ & $x$ & $x$ & $x$ & & $x$ \\
\hline 12 & 2000 & ECLSS & Doll and Eckart & $x$ & $x$ & $x$ & $x$ & $x$ & \\
\hline & & & \# of 12 times included & 12 & 8 & 12 & 10 & 7 & 7 \\
\hline
\end{tabular}

Equivalent mass always includes the equipment mass and the equivalent mass of the power supply. Volume and cooling usually are included and probably should be. The volume penalty was not included in four studies. The volume penalty may not be significant. It is highly correlated with mass, so it may not influence system comparisons. The cooling penalty was not included in two studies. Cooling load is often identical to the power requirement, so the power and cooling penalties are highly correlated. Volume and cooling have not been omitted in the six studies made since 1995. Logistics, the consumables and expendables, are not always mentioned but they indisputably contribute to launch mass. It is traditional and generally accepted that equivalent mass includes mass, volume, power, cooling, and logistics.
Including crew time is not traditional. Crew time was first included in equivalent mass by Drysdale et al. in 1992. His equivalent mass concept was not changed but was renamed ESM in 1998, with the establishment of the NASA ALS metric. Crew time is included in equivalent mass or ESM in all the seven papers by Drysdale and his co-authors. It is not in any of the other papers. More recently Doll and Eckart specifically stated that equivalent mass does not account for maintenance crew time.

Equivalent mass usually includes mass, volume, power, cooling, and logistics. It has not traditionally included crew time. The inclusion of crew time in ESM causes significant difficulties, as discussed in Appendix B. 


\section{APPENDIX B: EQUIVALENT MASS OF CREW TIME}

SUMMARY - This appendix reviews the two methods used in ALS to compute the equivalent mass of crew time and shows they give the same result for low amounts of crew time. An alternate derivation using a different justification gives the same formula. Several problems with the equivalent mass of crew time are discussed. It is shown that the equivalent mass of crew time is not an actual additional physical mass. It is a penalty mass that involves double counting some selected actual mass. A method is presented that avoids double counting of mass or cost. The mass equivalent of crew time is a cost benefit ratio that could be used as a metric.

COMPUTATIONS USED IN ALS - What is the cost of crew time in terms of mass? The basic approach is to identify some particular mass as the mass required to provide the crew time and then compute the mass equivalent of crew time, me(ct). A common assumption is that the mass necessary to provide all the crew time is the equivalent mass of the life support system. Since this mass is also included in traditional equivalent mass, allocating an additional life support system equivalent mass depending on crew maintenance time is a double charge, a penalty mass rather than an allocation of a real physical mass.

The ALS program uses two related approaches to compute the equivalent mass penalty for operations and maintenance crew time. Both are described in the Advanced Life Support Baseline Values and Assumptions Document (BVAD 2002) (References are in the references section of the main body.) The first method was defined by Levri et al::

"The key assumption used to determine the crew-time cost factor is as follows: Time occupied in operation and maintenance of the LSS (Life Support System) takes away from time for useful work, and the crew size and its associated LSS would need to be augmented to maintain the amount of useful time on the mission. For example, if half of the crew's work time were taken up by LSS maintenance and repair, then in order to accomplish the goals of the mission two identical systems and crews would have to be sent. This would double the ESM. Likewise, if three-fourths of the crew's available work time were consumed by the LSS, four identical systems and crews would need to be sent in order to accomplish the goals of the mission, quadrupling the ESM. Thus, the total ESM of the system is simply the ESM for mass, volume, power and cooling, multiplied by the ratio of work hours available to useful work hours (work other than LSS work)." (Levri et al. 2000)

In our notation the equivalent mass including mass, volume, power, cooling, and logistics is $\operatorname{EM}(m, v, p, c, l)$. The Equivalent System Mass (ESM) including equivalent mass (EM) plus crew time equivalent mass $E M(c t)$ will be designated as $\operatorname{ESM}(E M, c t)$. The approach of Levri et al. above is represented as follows:

$\operatorname{ESM}(E M, c t)=E M(m, v, p, c, l) *[$ total ct/(total ct - LSS ct)]

$=E M(m, v, p, c, l)+E M(m, v, p, c, l){ }^{*}[L S S$ ct $/($ total ct LSS ct)]

The first term is the traditional equivalent mass and the second term is the crew time penalty. As Levri et al. observe, the crew time penalty and the total ESM approach infinity as the time needed to operate and maintain the LSS (LSS ct) approaches the total crew time available (total ct). "This approach is considered to be adequate when the crew time requirement is a small percentage of the total crew time available." (Drysdale and Hanford 1999)

The second approach assigns a fixed cost rather than an increasing cost per crew hour. (BVAD 2002) (Jones 2001) The fixed cost method uses a constant mass equivalent of crew time. (This is similar to the fixed mass equivalent used for the volume, power, and cooling factors in equivalent mass.) Given the equivalent mass for the life support system and the total crew time, we divide the equivalent mass by the crew time to determine the mass-equivalent of crew time, me(ct), so many kilograms per hour.

$m e(c t)=E M(m, v, p, c, l) /$ total $c t$

Multiplying the crew time required by the life support system, LSS ct, by the mass-equivalent of crew time, me(ct), we obtain the equivalent mass of the life support system's crew time.

$E M(c t)=\mathrm{LSS} \mathrm{ct}^{*} \mathrm{me}(\mathrm{ct})$

ESM is the traditional equivalent mass plus the crew time equivalent mass penalty,

$E S M(E M, c t)=E M(m, v, p, c, I)+E M(c t)$

$=E M(m, v, p, c, l)+$ LSS ct * me(ct)

$=E M(m, v, p, c, l)+E M(m, v, p, c, l) *[L S S$ ct $/$ total ct $]$

The fixed cost approach is identical to the increasing cost approach of Levri et al. except that the denominator of the crew time penalty term is "total ct" rather than "total ct - LSS ct," The fixed cost and increasing cost ESM's are very close when "LSS ct" is much less than "total ct," which is the suggested area of application of the Levri et al. approach. (Drysdale and Hanford 1999) The maximum crew time penalty for "LSS ct" approaching "total ct" for the fixed cost approach is equal to the traditional equivalent mass. The maximum crew time penalty becomes infinite for the increasing cost approach. 
Drysdale and Hanford suggest using the increasing cost Levri et al. formula only when the crew time for LSS is a small percentage of total crew time, and using the fixed cost approach otherwise. This avoids the potentially infinite mass penality for crew time. However, the increasing cost Levri et al. formula is strictly correct, given its assumptions, whether LSS crew time is a large or small fraction of total crew time. The fixed-cost approach gives similar results only when the crew time requirement for LSS is a small percentage of total crew time.

The interpretation of the mass equivalent of crew time is as described by Levri et al. in the passage quoted above. We start with a life support system that has a certain equivalent mass and provides a certain total crew time. We assume that all this crew time is intended for "useful work hours (work other than LSS work)." Suppose in the extreme case that instead all the total crew time must be used for LSS work. Then we send a second mission with a second crew and second life support system to do the useful work. But again all the second crew's time must be used for LSS work, and so on until we send an infinite number of missions. This justifies the infinite mass penalty in the increasing cost approach of Levri et al.

What is the interpretation in the other extreme when a very small amount of the total crew time must be used for LSS work? Then we increase the size of the mission and the crew and the life support system proportionally, as [1 + LSS ct / total ct]. This justifies the fixed cost approach above. The fixed cost and increasing cost approaches give similar results for small added amounts of crew time.

ALTERNATE DERIVATION OF THE EQUIVALENT MASS OF CPEW TIME - These arguments are not completely convincing. The crew size and total crew work time are fixed mission parameters. The crew size can not be doubled and tripled. The crew size can not be increased by small fractional persons. A different justification based on the cost benefit ratio can be used to reach the variable cost formula of Levri et al. We start with a life support s;stem that has a certain equivalent mass and provides a certain total crew time. The total crew time is intende:! for exploration and science and for operations and maintenance. We define the total exploration and scionce crew time, es ct, as the benefit of the mission. We assume as above that the cost of crew time is equal to the traditional equivalent mass of the life support system, EM ( $m, v, p, c, I)$.

The initial cost/benefit ratio is

Cost/benefit $=E M(r, v, p, c, 1) /$ esct

If the benefit of exiloration and science crew time is reduced by an increesed requirement for operations and maintenance time, $\therefore \mathrm{ct}$, the cost benefit ratio increases to

Increased cost/benelt $=\operatorname{EM}(m, v, p, c, 1) /[$ es ct $-\Delta c t]$
To return to the more favorable original cost/benefit ratio, the equivalent mass would have to be decreased by some amount, $\triangle E M$. The decrease in equivalent mass required to obtain the same cost/benefit ratio can be regarded as the fair value of the decreased exploration and science crew time. If we could reduce equivalent mass this amount, we would have a comparable mission, one with the same cost/benefit ratio and the same crew size, but equally reduced cost and benefit. The reduced equivalent mass can be computed by equating the initial and final cost/benefit ratios.

Cost $/$ benefit $=\mathrm{EM}(\mathbf{m}, \mathbf{v}, \mathbf{p}, \mathbf{c}, \mathbf{l}) /$ es ct

$$
=[E M(m, v, p, c, l)-\Delta E M] /[\text { es ct }-\Delta c t]
$$

Manipulating (and simplifying the symbols) we have

$E / c=(E-d E) /(c-d c)$

$E / c=E / c(1-d E / E) /(1-d c / c)$

$1-\mathrm{dc} / \mathrm{c}=1-\mathrm{dE} / \mathrm{E}$

$d E / E=d c / c$

$\Delta E M / E M(m, v, p, c, l)=\Delta c t /$ es ct

The equivalent mass penalty is

$\Delta E M=E M(m, v, p, c, l) *[\Delta c t / e s c t]$

The $\operatorname{ESM}(E M, c t)$ is the sum of the traditional equivalent mass and the crew time penalty,

$\operatorname{ESM}(E M, c t)=E M(\mathbf{m}, \mathbf{v}, \mathbf{p}, \mathbf{c}, \mathbf{l})+\operatorname{EM}(\mathbf{m}, \mathbf{v}, \mathbf{p}, \mathbf{c}, \mathbf{l}){ }^{\star}[\Delta \mathbf{c t}$ / es ct]

Since $\Delta \mathrm{ct}=\mathrm{LSS} \mathrm{ct}$, and es ct $=$ total ct - LSS ct, this is the same as the increasing cost approach of Levri et al. If we accept that a mass penalty should be assigned for the use of crew time and should be added to traditional equivalent mass, the formula of Levri et al., rederived here, seems the best way to do it.

\section{PROBLEMS WITH THE EQUIVALENT MASS OF CREW} TIME -

Problem 1. Identifying the mass required to provide all the crew time is difficult. Above we used the equivalent mass of the life support system. It is as often assumed that the entire launch mass is required to provide the crew time.

"Most of the mass of a human space mission is needed to transport and support the crew, so the total mass and cost are approximately proportional to the number of crew (ignoring economies of scale). If we assume that the main purpose of the mission is to provide crew time, the total system mass is the appropriate cost for all the crew time." (Jones 2001) 
An early paper by Dysdale et al. (1992) divided launch cost by crew time to get cost per hour, divided launch mass by launch cost to get kilograms launched per dollar, then multiplied these numbers to get kilograms per hour, the mass equivalent of crew time. This is equivalent to dividing launch mass by crew time, so that the entire launch mass is assumed to be needed to provide the crew time. The total launch mass might be three times the life support mass, so this uncertainty is significant. (Jones 2001) Only the exploration and science research equipment is clearly not needed to deliver and support the crew.

Problem 2. Conversely, it can be argued that providing crew time on site for science and exploration is not the only or even the main purpose of the mission. This may be the case "for some types of missions (flag planting) and for some phases of a mission (possibly large portions of the transit legs to and from Mars)." (Levri et al. 2000) Ensuring the health and safoty of the crew is the most important objective. Allocating crew time to maintain life support should not be discouraged by unrealistic mass penalties on crew time use.

"On some missions the crew time needed may be less than the hours available, and then it is not appropriate to charge the actual cost. The crew needs important interesting work. ... The objective of assigning a cost to crew time should be to ensure the best use of the available time, and encourage the appropriate use of labor saving equipment in both mission science and crew support. We shouid not necessarily minimize the total use of crew time or direct it from crew support to science." (Jones 2001)

A major reason not to combine crew time with traditional equivalent mass in ESM is that this distorts the optimal allocation of crew tim?.

Problem 3. The assigned equivalent mass of crew time can not be checked in the same way as the factors in traditional equivalent mass. The actual hardware and launch mass that provide pressurized volume, power, and cooling can be readily identified. Crew time on site is not provided by purpose-built, separable, incrementally scalable hardware Orew time does "require" mass, volume, etc., but it does not physically "have" mass, volume, etc. Anything you could name requires some physical mass basis but many things are not in themselves physical objects. All the factors in traditional equivalent mass are based on hardware or material having part numbers. drewings, specifications, and test measurements incluting mass, volume, etc. A major reason not to combine crew time with power, volume, and cooling in ESI: is simply that crew time is not supplied by a limite: set of hardware on the launch manifest. Crex. tine! =s no srecific identifiable mass.

Problem 4. The ef: went mass of crew time is a prnalty imposed in addition to the actual anticipated f Inch mass. The penalty is proportional to some actual mass but can be infinitely large. The penalty is a hypothetical cost that does not correspond to any reasonable mission design change. The actual crew size, mission duration, and total crew hours are fixed mission design parameters. Crew size can not be increased by fractional or whole persons to provide more operations time. A major reason not to combine crew time with traditional equivalent mass in ESM is that the crew time penalty does not represent expected launch mass. It is a temporary accounting device intended to influence the design process, not an prediction of the result of the process.

AVOIDING DOUBLE COUNTING OF MASS - There is a way to combine mass-based cost and crew time-based cost without double counting. One solution is to assign some fraction of the launch cost to launch mass and the rest to crew time. Suppose that the fraction of the launch cost assigned to launch mass is flm. Then the fraction assigned to crew time is $(1-\mathrm{flm})$. The total launch cost is $L$, the total payload mass is $M$, and the total available crew time is CT. The launch cost assigned to a unit mass is $\mathrm{flm}{ }^{*} \mathrm{~L} / \mathrm{M}$. The launch cost assigned to an hour of crew time is $(1-f \mid m)^{*} \mathrm{~L} / \mathrm{CT}$.

Suppose a system has some mass, $\mathbf{m}$, and some crew time, ct. The total mass and crew time launch cost assigned to this system is $m^{*} \mathrm{flm} \mathbf{m}^{*} \mathrm{~L} / \mathrm{M}+\mathrm{ct}^{*}(1-\mathrm{flm})^{\star} \mathrm{L} / \mathrm{CT}$. If we sum this over all systems we get $M^{*}$ flm* $L M+C T^{*}(1$ - $f(\mathrm{~m})^{\star} \mathrm{L} / \mathrm{CT}=\mathrm{L}$.

This crew time penalty does not double charge. We should select some value for flm, to set the relative weights of mass and crew time. We can call this weighted mass and crew time penalty the mission cost metric. It is a specially designed system launch cost allocation depending on mass and crew time. It is measured not in kilograms but in some cost unit. Since $L$ is the base cost and appears in all terms of the metric, its units can be set arbitrarily.

THE MASS EQUIVALENT OF CREW TIME AS A METRIC - The mass equivalent of crew time, me(ct) in $\mathrm{kg} /$ hour, is constant for a given life support design. Suppose we have a human habitat with life support, crew, etc., ready for launch. If we launch it to LEO or even take it to the Moon or Mars, the mass and the science crew time are still the same, and so is their ratio. The mass equivalent of crew time is a cost-benefit ratio, the mass cost divided by the crew science hours benefit.

The mass equivalent of crew time, the mass cost/crew time benefit ratio, is actually a better metric than traditional equivalent mass or ESM. It can be optimized. Different systems with different equivalent mass cost and crew time benefit can be compared. Selecting technology to minimize the mass equivalent of crew time would accomplish the original objective of ESM, reducing mass and maximizing science time. 
APPENDIX C: WIAL TO PAYLOAD MACS RATIO INCLUDIN a THE ROCKET VEHICLES

The calculation beiow of the combined (product) initial/payload mass ratio in LEO considers the final payload landed or returned to LEO, the second stage landing or return rocket and propellant, and the first stage LEO to Moon or Mars orbit rocket and propellant. The mass ratios including the rocket vehicles are significantly higher than in tables 1 and 2 .

We define the variab's for the calculation as follows:

S: mass of the final payload landed or returned to LEO

R2: mass of the second stage landing or return rocket

P2: mass of the second stage landing or return propellant

R1: mass of the first stage LEO to Moon or Mars orbit rocket

P1: mass of the first stage $1=0$ to Moon or Mars orbit propellant

The initial/final mass ratios for the second stages (for deorbit and landing or for Moon or Mars orbit back to LEO) are 1.8.1.3, and 1.4 from tables 1 and 2, excent for Mars orbit back to LEO which is 3.5. We use 1.5 as the typical second stage nitial/final mass ratio and consider the case of 3.5 later.

We set $\mathbf{S}=1$. The vehicle mass is $15 \%$ of the propollant mass. (Larson and Franke 2000, p. 405) (Wertz and Larson 1996, p. 242) $R 2=0.15$ P2. Using the typical initial/final mass ratio of 1.5 for the second stage, $? \mathbf{2}=$
$1.5(\mathrm{~S}+\mathrm{R} 2)=1.5 * 1+1.5 * 0.15 \mathrm{P} 2$. We then solve for $\mathbf{P 2}=1.94, \mathbf{R} 2=0.29$, and $\mathbf{S}+\mathbf{P 2}+\mathbf{R 2}=3.23$. The typical second stage initial/final mass ratio of 1.5 becomes 3.23 when we consider the vehicle mass.

The initial/final mass ratios for the first stage (LEO to Moon or Mars orbit) are 3.7 and 3.8 from tables 1 and 2 . As for the second stage, $\mathbf{S 1}=3.23, \mathbf{R 1}=0.15 \mathbf{P 1}, \mathbf{P} 1$ $=3.7(\mathrm{~S} 1+\mathrm{R} 1)=3.7^{*} 3.23+3.7 \cdot 0.15 \mathbf{P 1}$. P1 $=26.8$. $\mathbf{R} 1=4.02 . \mathbf{S} 1+\mathbf{R} 2+\mathbf{P} \mathbf{2}=34.05$. The typical first stage initial/final mass ratio of 3.7 becomes 10.5 when we consider the vehicle mass. The combined (product) initial/payload mass ratio increases from $1.5 \cdot 3.7=5.6$ to 34.0 .

It is usual to reduce such large mass ratios by using additional rocket staging. Using two stages for one original stage reduces the mass ratio by about 25 percent. (Larson and Pranke 2000, pp. 776-7) The final mass ratio is $0.75 * 3.23 \cdot 0.75 \cdot 10.54=19.1$. For a Moon landing, a return from Moon or Mars orbit to LEO, or a Mars landing, the initial/payload mass ratio is roughly 20.

An exactly similar calculation is made for Mars orbit back to LEO. The initial/final mass ratios for the second stages for Mars orbit back to LEO is $3.5 . \mathbf{S}=1 . \mathbf{R 2}=0.15 \mathbf{P 2}$, $\mathbf{P 2}=3.5(\mathrm{~S}+\mathrm{R} 2)=3.5^{*} 1+3.5{ }^{*} 0.15 \mathbf{P 2} . \mathbf{P 2}=7.37$, $\mathbf{R} 2=1.1$, and $\mathbf{S}+\mathbf{P} \mathbf{2}+\mathbf{R} \mathbf{2}=9.47$. The second stage initial/final mass ratio of 3.5 becomes 9.47 when we consider the vehicle mass. For the first stage (LEO to Mars orbit), S1 $=9.47, \mathbf{R} 1=0.15 \mathbf{P 1}, \mathbf{P 1}=3.7(\mathbf{S} 1+$ R1) $=3.7^{\star} 9.47+3.7 \cdot 0.15$ P1. P1 $=78.7 . \quad$ R1 $=11.8$. $\mathbf{S 1}+\mathrm{R2}+\mathrm{P2}=100.0$. Using two stages for each original stage reduces this to about 50 . 